\title{
FUSE binding protein FUBP3 is a potent regulator in Japanese encephalitis virus infection
}

\author{
Peng $\mathrm{Xu}^{1}$, Wei Tong ${ }^{2}$ and Young-Mao Chen ${ }^{3,4^{*}}$ (i)
}

\begin{abstract}
Background: The JEV genome is a positive-sense RNA with a highly structured capped $5^{\prime} U T R, 3^{\prime} U T R$ and a large open reading frame. $3^{\prime} U T R$ is the untranslated region of flavivirus and has various important functions during viral replication, such as translation, replication and encapsidation. During viral replication, the $3^{\prime} U T R$ interacts with viral proteins and host proteins and is required for viral RNA replication and translocation.

Methods: The expression level of FUBP3 was knocked down by siRNA and Flag-tagged FUBP3 overexpression plasmid was constructed for overexpression. BHK-21 cells were cultured and infected with JEV to investigate the functional role of FUBP3 in the viral infection cycle. Subcellular localization of FUBP3 and viral replication complexes was observed by dual immunofluorescence staining.

Results: Four host proteins were specifically associated with the $3^{\prime}$ UTR of JEV, and FUBP3 was selected to further investigate its potential functional role in the JEV infection cycle. Knockdown of FUBP3 protein resulted in a significant decrease in JEV viral titer, whereas ectopic overexpression of FUBP3 resulted in increased JE viral infectivity. In cells stably knocked down for FUBP3 and then infected with JEV, we found almost no detectable viral NS5 protein. In contrast, when cells stably knocking-down of FUBP3 overexpressed FUBP3, we found a significant increase in viral RNA production over time compared to controls. We also demonstrated that FUBP3 re-localized in the cytoplasm after infection with JEV and co-localized with viral proteins. Exogenous overexpression of FUBP3 was also shown to be located in the JE replication complex and to assist viral replication after JEV infection.
\end{abstract}

Conclusions: The overall results suggest that FUBP3 regulates RNA replication of JEV and promotes subsequent viral translation and viral particle production.

Keywords: Japanese encephalitis virus, Far upstream element-binding protein 3, Untranslated region, Viral replication

\section{Introduction}

Japanese encephalitis (JE) is an infectious disease caused by a Japanese encephalitis virus (JEV) that infects the central nervous system of humans. Transmission of Japanese encephalitis virus is primarily carried by mosquitoes as vectors and can be transmitted between humans,

\footnotetext{
*Correspondence: ymc868@mail.ntou.edu.tw

3 Bachelor Degree Program in Marine Biotechnology, College of Life Sciences, National Taiwan Ocean University, Keelung 20224, Taiwan Full list of author information is available at the end of the article
}

birds, pigs, bats, and horses, as well as other animals. The incubation period of Japanese encephalitis virus is 4-14 days, and clinical symptoms include fever, headache, vomiting, loss of appetite, and mental disturbance; in severe cases, fever, headache, vomiting, and neurological symptoms appear suddenly, while neurological symptoms include seizures, tremors, photophobia, and limb disorders, and the most severe cases can lead to coma or death [1].

The JEV genome is a single-stranded and positivesense RNA with a genome size of 10,976 nucleotides.

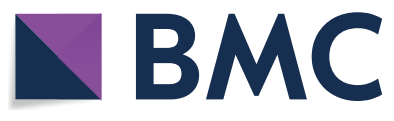

(c) The Author(s) 2021. Open Access This article is licensed under a Creative Commons Attribution 4.0 International License, which permits use, sharing, adaptation, distribution and reproduction in any medium or format, as long as you give appropriate credit to the original author(s) and the source, provide a link to the Creative Commons licence, and indicate if changes were made. The images or other third party material in this article are included in the article's Creative Commons licence, unless indicated otherwise in a credit line to the material. If material is not included in the article's Creative Commons licence and your intended use is not permitted by statutory regulation or exceeds the permitted use, you will need to obtain permission directly from the copyright holder. To view a copy of this licence, visit http://creativecommons.org/licenses/by/4.0/. The Creative Commons Public Domain Dedication waiver (http://creativeco mmons.org/publicdomain/zero/1.0/) applies to the data made available in this article, unless otherwise stated in a credit line to the data. 
JEV genomic RNA consists of a highly structured capped $5^{\prime}$ UTR, a $3^{\prime}$ UTR and a large open reading frame (ORF) $[2,3]$. When JEV enters the host cell, it releases viral RNA and translates one polyprotein, which is then cleaved by viral NS2B/3 protease and host proteases to obtain the viral structural and nonstructural proteins. Structural proteins are divided into outer capsid proteins, membrane protein precursors and an envelope protein; nonstructural proteins are divided into NS1, NS2A, NS2B, NS3, NS4A, NS4B, NS5, which play important roles in viral infection $[2,4-6]$.

The $3^{\prime}$ UTR is the untranslated region of flaviviruses and has various important functions during viral replication, such as translation, replication and encapsidation [7, 8]. This $3^{\prime}$ UTR contains several functional motifs, including conserved sequences (CS motifs), cyclization motifs, pseudoknot structure and $3^{\prime}$-stemloop ( $3^{\prime}$-SL) motif. Past studies have identified two pairs of cyclization motifs in which the $5^{\prime}$ and $3^{\prime}$ conserved sequences complement to each other and may form the cyclization structure [9]. The upstream of the AUG codon ( $\left.5^{\prime} \mathrm{UAR}\right)$ is located in the second pair of cyclization motifs, which is complementary to the sequence located in the $3^{\prime} \mathrm{UTR}$ ( $\left.3^{\prime} \mathrm{UAR}\right)$. The replication of DENV and WNV confirmed the necessity of the existence of the UAR cyclization motif [10]. In addition to this, another element located downstream of the AUG (designated as $5^{\prime} \mathrm{DAR}$ ) has been shown to be involved in the cyclization of the genome during the replication of DENV [11]. It has also been reported that the $3^{\prime} \mathrm{UTR}$ of JEV is a structural domain of six components [12]. Two of these $3^{\prime}$-proximal structural domains are sufficient for the replication of viral RNA [13]. In addition to RNA-RNA interactions within the viral genome, the $3^{\prime}$ UTR of JEV interacts with viral proteins and host proteins that are required for viral RNA replication and translocation [14].

The far upstream element-binding proteins (FUBP) comprise a three-gene family whose physiological functions are primarily single-stranded nucleic acid binding proteins. FBP (encoded by FUBP1), the 2 other proteins, including FUBP2 encoded by KHSRP and FBP3 encoded by FUBP3 [15]. Several studies have shown that FUBPs interact with many DNA or RNA targets in the cells and are further involved in various biological processes, including RNA transport, translational regulation, mRNA degradation, and transcription [16-18]. The structure of FUBPs includes a central structural domain consisting of four regularly spaced $\mathrm{K}$ homology $(\mathrm{KH})$ patterns that recognize similar sequences in singlestranded DNA or RNA targets [19]. As confirmed by various in vitro and tissue culture experiments, FUBPs bind to complex DNA and RNA targets. Many neoplastic diseases are closely associated with dysregulation of FUBPs. Although high levels of FUBP expression are associated with poor tumor growth and prognosis in hepatocellular carcinoma, non-small cell lung cancers and gliomas, FUBP expression accounts for a significant proportion of oligodendrogliomas, where it is a tumor suppressor. FUBP1 is widely expressed in a number of tissues and cells with a distinct spatial-temporal profile, and it affects a number of cellular processes through the effects of transcription, mRNA stability, and translation, including differentiation, cell proliferation, apoptosis or cell death [20-24]. Aberrant expression of this gene has been found in malignant tissues, and the gene is important for neural system and lung development. The binding of this protein to viral RNA is thought to play a role in several viral diseases, including hepatitis $C$ and handfoot-mouth disease. FUBP3 is a single-stranded DNAbinding protein that recognizes FUSE. The gene encoding FUBP3 is located on chromosomes 9q34.1 [25, 26]. Studies have shown that FUBP3 provides substantial support for the proposed role of FUBP1 and FUBP3 as activators of c-myc in vivo [27]. In addition, FUBP3 was recently reported to interact with the $5^{\prime}$ untranslated region of enterovirus 71 (EV-A71) to regulate its replication in differentiated neuronal cells [28].

In the present study, we identified four host proteins specificities associated with the JEV $3^{\prime}$ UTR, and FUBP3 and FUBP1 were among them. Since FUBP1 has been reported to negatively regulate the infection cycle of JEV [29], this study focused on the possible role of FUBP3 in the JEV infection cycle.

\section{Methods}

\section{Cell culture and viruses}

We used RPMI 1640 medium (Gibco-Invitrogen, Carlsbad, CA, USA), cultured Baby Hamster Kidney-21 (BHK21) cells supplemented with $2 \%$ fetal bovine serum (FBS) (Gibco-BRL, Carlsbad, CA, USA) and $24 \mathrm{mM}$ sodium bicarbonate (Sigma, St. Louis, USA), and maintained at $37{ }^{\circ} \mathrm{C}$ in an atmosphere of $5 \% \mathrm{CO}_{2}$. Virus stocks were obtained from Dr. WJ Chen and referred to as T1P1 strain (accession number: AF254453). For viral propagation, we infected the BHK-21 cells at a multiplicity of infection (MOI) of 2. BHK-21 cells infected with JEV were then cultured for 2 days before being harvested for further experiments.

\section{RNA pull-down experiments and LC-MS/MS analysis}

The construction of plasmid pJEV-3'UTR was previously described [30]. The $3^{\prime}$ UTR fragment flanked by BamHI sites was excised from the pUC19 vector, and in vitro RNA transcripts were obtained using the HiScribeTM T7 in vitro transcription kit (New England bioLabs). Biotinylated RNA was synthesized in a mixture containing 
NTP, bio-labeled UTP, reaction solution and other reagents according to the kit protocol. The non-biotinylated RNA was synthesized in the same reaction mixture without biotin-labeled UTP. After in vitro transcription, we used the RNeasy protect mini kit to purify the newly synthesized RNAs for the subsequent experiments. The synthesize RNAs were incubated with BHK-21 cell lysates for $4 \mathrm{~h}$, streptavidin beads were then applied to capture the proteins associated with the biotinylated JEV $3^{\prime}$ UTR. Proteins pulled down from cell lysates were separated using 8\% SDS-PAGE and silver stained [30]. Four selected gel bands were subjected to in-gel tryptic digestion and the digested peptides were extracted with acetonitrile and dried in a SpeedVac for the LC-MS/MS analysis.

\section{Reverse-phase LC-MS/MS analysis}

The standard procedure of LC-MS/MS was described previously [28]. In brief, the trypsin digested peptides were reconstituted in buffer containing $0.1 \%$ formic acid and loaded onto a trap column (Zorbax 300SB-C18, $0.3 \times 5 \mathrm{~mm}$; Agilent Technologies, Taiwan) at a flow rate of $0.2 \mu \mathrm{L} / \mathrm{min}$ in HPLC; the peptides were separated on a resolving analytical C18 column (New Objective, Woburn, MA, USA). The peptides were eluted using a linear gradient of $0-10 \%$ buffer in $99.9 \%$ acetonitrile containing $0.1 \%$ formic acid. The liquid chromatograph was then connected to a two-dimensional linear ion trap mass spectrometer (LTQ-Orbitrap, Thermo Fisher, CA, USA). For the MS analysis, we used a data-dependent procedure alternating one mass spectrometry scan and six MS/ MS scans for the six most abundant precursor ions.

\section{Construction of flag-tagged FUBP3 expression plasmid}

To generate the $3 \times$ Flag-FUBP3 plasmid, we RT-PCR amplified the full-length of FUBP3 from the RNA isolated from BHK-21 cells using the forward primer, 5'-GCGATATCATTCGGCTCCTGAAGCACC-3', and the reverse primer, $5^{\prime}$-GTTTAAGCCGTAGAATCG TCCATGCG-3'. The PCR product was then treated with $E c o R V$ and $X b a \mathrm{I}$, and was ligated into the $\mathrm{p} 3 \times$ Flag-mycCMV vector that was previously digested with EcoRV and $X b a \mathrm{I}$. The constructed plasmid was verified by sequencing. To overexpress the FUBP3 recombinant protein, cells were transfected with $2.5 \mu \mathrm{g}$ of plasmid DNA using lipofectamine 3000 (Invitrogen) according to the product manual.

\section{RNA preparation and real-time PCR}

RNA extraction was performed as described [31]. Briefly, viral RNA was prepared using QIAamp ${ }^{\circledR}$ viral RNA mini kit (Qiagen, Hilden, Germany). JEV specific singlestranded cDNA was synthesized from $3 \mu \mathrm{g}$ of cytoplasmic RNA of JEV-infected BHK-21 cells at 2 days post infection. Quantifications of the JE viral RNA were performed by quantitative real time PCR (qPCR) using the orward and reverse primers, GTTTTGGGAGCCTTA CTTGT and GCTAAGCATGTTCATCACTA, respectively. The qPCR analyses were performed in duplicate using SYBR green master mix (KAPA) in an ABI 7500 qPCR system.

\section{Knockdown of FUBP3}

We carried out two methods to knockdown the expression levels of FUBP3 in BHK-21 cells. (1) siRNA transfection: FUBP3 siRNA (5'-GUGUCGAGUAGCUAGC-3') were synthesized by MDBio Inc. Scrambled siRNA was designed and synthesized by MDbio (New Taipei City, Taiwan). The siRNA was transfected into cells using RNAiMAX lipofectamine (Invitrogen) in Opti-MEM reduced serum medium (Invitrogen). The siRNA was incubated with RNAiMAX for $30 \mathrm{~min}$ at room temperature prior to transfection, and FUBP3 expression was silenced during 2-day incubation as determined by Western blotting. (2) shRNA transfection: Plasmids containing fubp3 short hairpin RNA (shRNA), pLKO-fubp-3 shRNA (pFubp-3i: GTGTCGAGTAGCTAGC), and a negative control, luciferase shRNA (pNCi: GTACGC GGAATACTTCGA), were obtained from the National RNAi Core Facility, Academia Sinica, Taiwan. BHK-21 cells transfected with pFubp-3 or pNCi were selected with puromycin.

\section{Western blotting}

For Western blotting, an equal amount of cell lysates was denatured for $5 \mathrm{~min}$, separated by 12\% SDS-PAGE under reducing conditions, and then electro-transferred to a methanol-activated polyvinylidene difluoride (PVDF) membrane (Bio-Rad Laboratories, Hercules, CA). The membrane was blocked with $5 \%(\mathrm{wt} / \mathrm{vol})$ nonfat dried milk in PBS-T buffer (20 mM sodium phosphate pH7.4, $137 \mathrm{mM} \mathrm{NaCl}$, and $0.1 \%$ Tween 20 ) at room temperature for $30 \mathrm{~min}$, followed by incubation with mouse anti-FUBP1 and anti-FUBP3 antibodies (Bioworld, Minnesota, USA); rabbit anti-NS5 (Yao-Hong Biotechnology Inc, New Taipei city, Taiwan) and secondary antibodies with an HRP-conjugated goat anti-mouse or anti-rabbit IgG (Sigma, St. Louis, USA), at a 1:10,000 dilution in PBS-T buffer containing $0.5 \%$ nonfat milk at room temperature for $1 \mathrm{~h}$. Following three time washes with PBS-T buffer the membrane was developed by ECL (Millipore, MA, USA).

\section{Viral plaque assay}

The standard viral plaque assay was described elsewhere [30]. In brief, a serial tenfold dilutions of the supernatant of JEV infected medium were prepared and infected on 
the one-layer of BHK-21 cells. After 1-h infection, the medium was removed and cells were washed twice with PBS to remove the unbound viruses. Next, we added $2 \mathrm{~mL}$ of RPMI 1640 medium containing 5\% FBS and 0.3\% seaplaque agarose (Invitrogen, Carlsbad, CA) to each well. The 6 well TC plates were then incubated at $37{ }^{\circ} \mathrm{C}$ for at least 4 days, followed by fixing with $2 \mathrm{~mL}$ of $10 \%$ formaldehyde, and kept for $30 \mathrm{~min}$ at room temperature $\left(22-25{ }^{\circ} \mathrm{C}\right)$ before removal of $0.3 \%$ agarose. The monolayer cells were stained with crystal violet stain solution (0.5\% crystal violet, $1.85 \%$ Formalin, $50 \% \mathrm{EtOH}, 0.85 \%$ $\mathrm{NaCl}$ ) (Sigma) and calculated plaque-forming units (pfu/ $\mathrm{mL}$ ) with the virus titer formula, where virus titer equals the number of plaque $\times(1 \mathrm{~mL} / 0.5 \mathrm{~mL}) \times$ dilution factor.

\section{Immunofluorescence and antisera}

BHK-21 cells were cultured on glass coverslips for immunofluorescent staining. After infection, the cells were rinsed with PBS and fixed with $4 \%$ paraformaldehyde in PBS for $30 \mathrm{~min}$ at room temperature. Prior to incubation with antibodies, the cells were permeabilized with $0.1 \%$ Triton X-100 in PBS for 30 min and incubated in F1 blocking solution (Biofuture biotech, Taoyuan, Taiwan) for $5 \mathrm{~min}$, followed by incubation sequentially with primary antibodies: mouse anti-FUBP3 protein (Bioworld, Minnesota, USA); rabbit anti-NS5 (Yao-Hong Biotechnology Inc, New Taipei city, Taiwan); rabbit anti-dsRNA (Bioworld, Minnesota, USA) and secondary antibodies: (conjugated with Texas Red) and (conjugated with FITC). After immunostaining, coverslips were mounted on slides in gelvatol medium. Images were acquired using a Zeiss confocal microscope (LSM 510) and processed with Adobe Photoshop software (Adobe, CA).

\section{Statistical methods}

All experiments were repeated at least 3 times. Values are given as means and standard errors of the mean (SEM). Data were analyzed using Graph Pad Prism 4.0 software. Statistical significance was assessed by Student's $t$-test or one-way ANOVA. $p$ values less than 0.05 were considered significant.

\section{Results}

\section{The FUBP3 protein interacted with the JEV $3^{\prime}$ UTR}

To identify host proteins bound to the JEV $3^{\prime}$ UTR, the RNA fragment was labeled with biotin and then incubated with BHK-21 cell lysates. After the reaction, streptavidin beads were applied to capture proteins associated with the biotinylated JEV $3^{\prime} U T R$. The biotinylated RNA-associated proteins were pull downed and then separated in SDS-PAGE and observed after silver staining (Fig. 1a). Four bands were observed associating with the biotinylated JEV 3'UTR (Fig. 1a, lane 4) compared to the non-biotinylated JEV 3'UTR (Fig. 1a lane 3). The proteins in these four bands were then subjected to in-gel trypsin digestion and subsequently identified by liquid chromatography tandem-mass spectrometry (LC-MS). For database searches, Mascot Server and Swiss-Prot database were used and further integrated using Scaffold software. The identified proteins were GRP78, FUBP1, FUBP3 and hnRNP A1. Interestingly, GRP78, a molecular chaperone, was among these proteins. GRP78 has been reported to be an important host factor involved in JEV in viral maturation and present in subsequent cellular infections [30]. In addition, we also noted that two far upstream element (FUSE) binding proteins, FUBP1 and FUBP3, were among these proteins. To further confirm the results of RNA pull-down, the biotinylated RNA-associated proteins were eluted for protein electrophoresis. Western blotting was performed to detect the presence of these two proteins using anit-FUBP1 and anti-FUBP3 antibodies (Fig. 1b). FUBP1 has been reported to interact with the JEV $3^{\prime}$ UTR and acted as a negative regulator in the JEV infection cycle [29]. Since FUBP3 has been reported to bind RNA and contain helicase activity on the RNARNA duplexes [32], we decide to further investigate whether FUBP3 has potential functional role(s) in the JEV infection cycle.

\section{FUBP3 affects JEV replication}

FUBP3 is a protein known to bind RNA and regulate the replication of certain viruses, so we transfected siRNA (siFUBP3) into BHK-21 cells for $24 \mathrm{~h}$ and then analyzed the protein expression of FUBP3, followed by infecting the cells with JEV $(\mathrm{MOI}=2)$. First, after transfection with siFUBP3 for $24 \mathrm{~h}, \mathrm{BHK}-21$ cells were still viable, as assessed by MTT assay (Fig. 2a). Next, transfection with siFUBP3 resulted in an approximately tenfold decrease in FUBP3 expression in the cells compared to the control (si-scramble) (Fig. 2b). As expected, the viral titers of JEV were significantly reduced by approximately twofold ( 24 h.p.i) and sixfold (48 h.p.i) compared with the control group (Fig. 2c). As shown above, a decrease in FUBP3 protein was found to cause a significant decrease in JEV viral titers; therefore, we wanted to test whether overexpression of FUBP3 in cells would increase JE viral titer. First, the pCMV-FUBP3-Flag vector was transfected into BHK-21 cells for $24 \mathrm{~h}$. FUBP3 protein expression and JEV viral titer were analyzed. As shown in Fig. 2d, we detected ectopic expression of FUBP3 in pCMVFUBP3-Flag-transfected BHK-21 cells. JE virus titers increased approximately threefold in cells in the FUBP3overexpressed cells $48 \mathrm{~h}$ after JEV infection compared to the mock and vector control groups (Fig. 2e). From the results, it appears that FUBP3 protein has the ability to regulate JE viral particles production. 
(A)

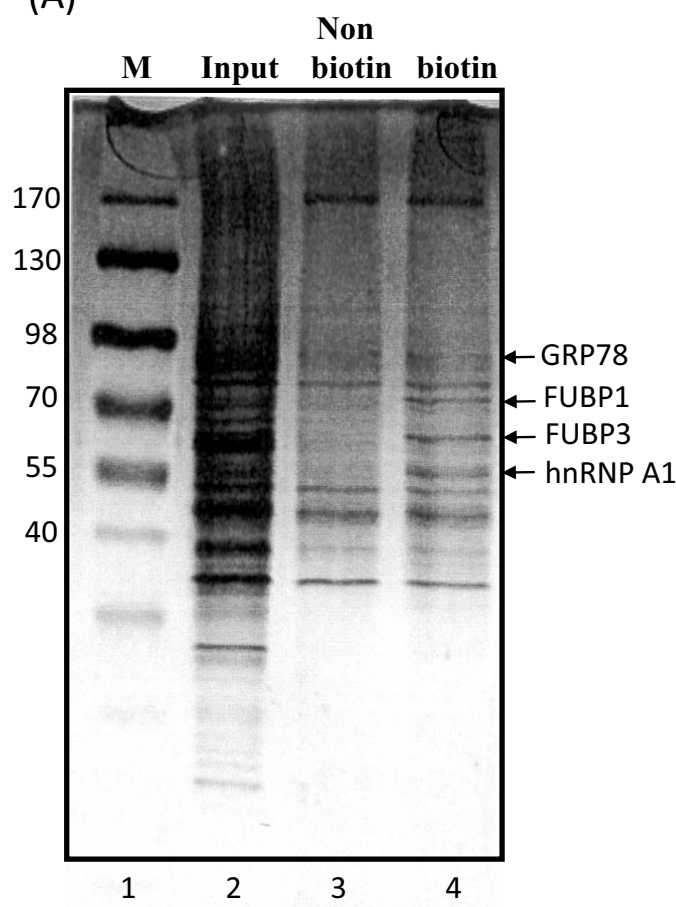

(B)

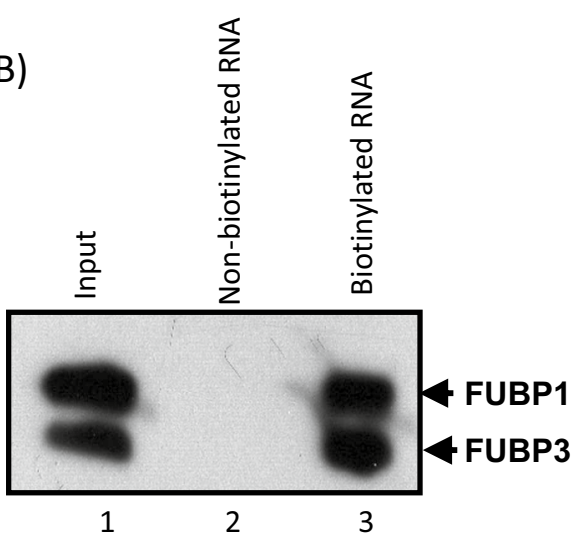

Fig. 1 Identification of host proteins associated with JEV 3'UTR. a A biotin-labeled JEV 3'UTR was incubated with BHK-21 cell lysates. Streptavidin was then applied to pull down the labeled RNA and associated host proteins. The eluates were subsequently subjected to SDS-PAGE analysis. Lane 1: protein size marker; lane 2: input of BHK-21 cell lysates; lane 3: BHK-21 cell lysates incubated with the non-biotinylated JEV 3'UTR; lane 4: BHK-21 cell lysates incubated with the biotinylated JEV 3'UTR. b Western blotting was performed, and both anti-FUBP3 and anti-FUBP1 antibodies were used to detect the presence of these two proteins

\section{JEV infectivity correlates with the presence of FUBP3}

Since the above results showed that FUBP3 increased JE viral particles production, we next investigated whether FUBP3 could also regulate the viral translation and replication during JEV infection. First, FUBP3 was stably knockdown expressed using the shFUBP3 plasmid, and we detected the decreased expression level of FUBP3 in sh-FUBP3-transfected cells compared to sh-luciferasetransfected and mock BHK-21 cells (Fig. 3a). BHK-21 cells transfected by shFUBP3 remained viable as assessed by the MTT assay in Fig. 2a above (data not shown). Next, cells were then infected with JEV $(\mathrm{MOI}=2)$ and cultured for $4,8,16,24,32,40$, and $48 \mathrm{~h}$. Viral protein expression was detected by Western blotting using anti-NS5 specific antibody. We found that viral NS5 protein was barely detected in the FUBP3 knocked down cells (Fig. 3b) compared to untreated cells (Fig. 3c), indicating that FUBP3 is an important regulator of JEV protein translation. As expected, the viral titers of JEV were significantly reduced by approximately fivefold (24 h.p.i) compared to the control group (Fig. 3d). Next, in the JEV RNA replication experiment, viral RNA production was analyzed by first stabilizing knockdown cells overexpressed with FUBP3 and then infecting them with JEV $(\mathrm{MOI}=2)$ for $4,8,16$, 24, 32, 40 and 48 h. As shown in Fig. 3e, when cells with stable knockdown of FUBP3 overexpressed FUBP3, we found a significant increase in viral RNA production over time compared to the control group. The overall results demonstrated that FUBP3 regulates the replication of JEV RNA replication and promoted subsequent viral translation and viral particle production.

\section{JEV infection redistribute the subcellular localization of FUBP3}

It has been shown that FUBP3 protein is present in the cytoplasm and nucleus of cells, and it has been suggested that some viral infections may lead to redistribution of host proteins in cells. In the above experimental results, we know that FUBP3 regulated the replication of JEV, which in turn regulates the subsequent viral translation and viral particles production. Therefore, we wanted to understand whether the endogenous FUBP3 protein redistributes after JEV infection and to confirm the colocalization with the JEV NS5 protein. For this purpose, 


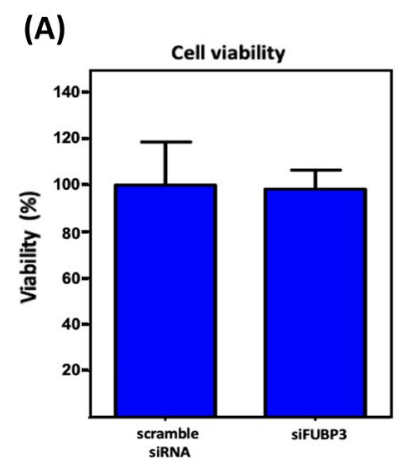

(D)

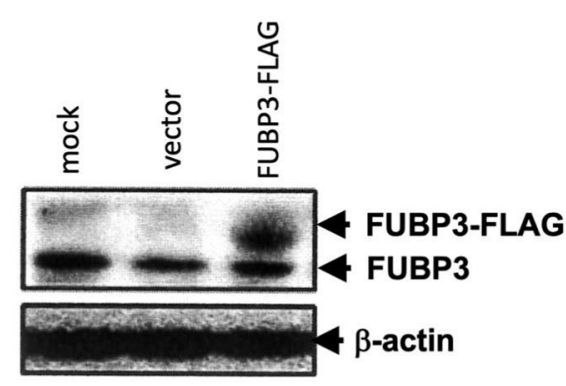

(B)

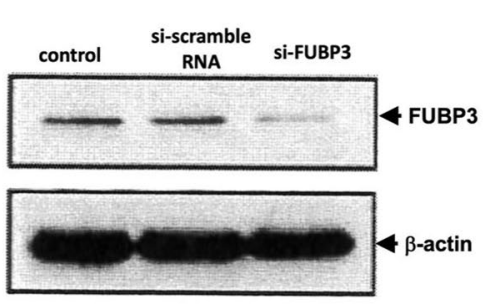

(C)

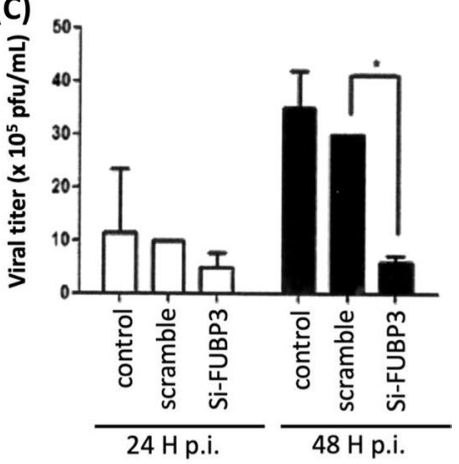

(E)

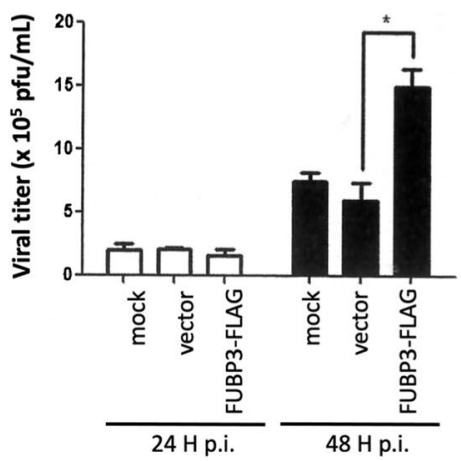

Fig. 2 Knockdown of FUBP3 reduces JEV infectivity. a BHK-21 cells were transfected with siFUBP3 (5 $\mu$ M) or si-scramble RNA (negative control), followed by culturing the cells for $24 \mathrm{~h}$ prior to the MTT assay. The si-scramble RNA-transfected cells were used as $100 \%$ of cell viability. The results shown here were the representative of three independent experiments. b BHK-21 cell lysates were harvested and protein expression was analyzed by Western blotting using anti-FUBP3 specific antibody. c The si-FUBP3-transfected-BHK-21 cells were then infected with JEV $(\mathrm{MOI}=2)$ for 24 and $48 \mathrm{~h}$, followed by analysis of viral titers using plaque forming assay $\left({ }^{*} p<0.05 ;{ }^{* *} p<0.01\right.$ ). $\mathbf{d}$ To overexpress the FUBP3, pCMV-8 vector (control) and pCMV-FUBP3-Flag vector $(8 \mu \mathrm{g})$ were transfected into BHK-21 cells for $24 \mathrm{~h}$. Protein expression was analyzed by Western blotting as described above. e FUBP3-overexpressed BHK-21 cells were then infected with JEV $(\mathrm{MOI}=2)$ for 24 and $48 \mathrm{~h}$, followed by analysis of viral titers using plaque forming assay $\left({ }^{*} p<0.05 ;{ }^{* *} p<0.01\right)$

BHK-21 cells were infected with JEV $(\mathrm{MOI}=2)$ for $48 \mathrm{~h}$ and the localization of FUBP3 and JEV NS5 viral proteins was analyzed by confocal microscopy. The results showed that FUBP3 and JEV NS5 viral proteins were co-localized in the cytoplasm (Fig. 4e, f) compared to the control (Fig. 4b, c). The Pearson correlation coefficient measures of images of green fluorescent protein FITC-FUBP3 and Texas Red-NS5 is 0.9998 . This implies that after JEV infection, FUBP3 was re-localized in the cytoplasm and co-localized with viral proteins. In addition, we overexpressed the FUBP3 protein in BHK-21 cells prior to JEV infection. As shown in Fig. 5b, the cytoplasm of overexpressed FUBP3 cells had many positive stains for FUBP3 (Fig. 5b), and after infection with JEV, the intracellular distribution of many FUBP3 (Fig. 5f) was affected by JEV and then co-localized with the viral NS5 protein (Fig. $5 \mathrm{~g}$ ), as shown around the viral replication sites (Fig. 5e). Additionally, the Pearson correlation coefficient measures of images of overexpressed FITC-FUBP3 and Texas RedNS5 is 0.9994, indicating that FUBP3 was co-lcocalized with viral NS5 proteins.

\section{FUBP3 was recruited to the viral replication complexes of JEV}

From the above results, it is clear that FUBP3 has a greater effect on JEV replication, as FUBP3 and JEV NS5 protein were found to co-localize in the cytoplasm, and JEV NS5 protein itself is an RNA dependent RNA polymerase responsible for viral replication. Therefore, we speculated whether FUBP3 would be transferred to the JE replication complex to promote viral replication after JEV infection. We infected BHK-21 cells with JEV $(\mathrm{MOI}=2)$ for $48 \mathrm{~h}$ and analyzed the localization of FUBP3 and viral double-strand RNA (dsRNA), which is present in the replication complex during replication, by confocal microscopy. The results showed that the co-localization of FUBP3 (Fig. 6g, k) with viral dsRNA (Fig. 6h, i) in the 
(A)

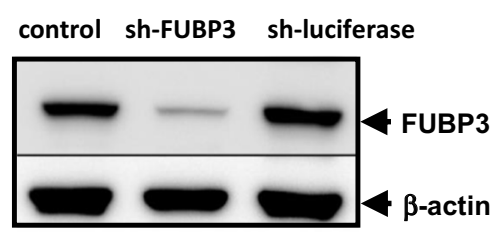

(B)

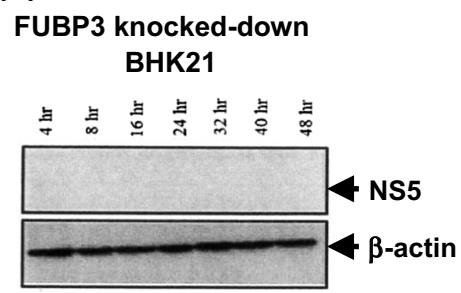

(C)

\section{Untreated BHK21}

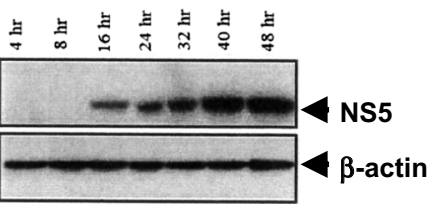

(D)
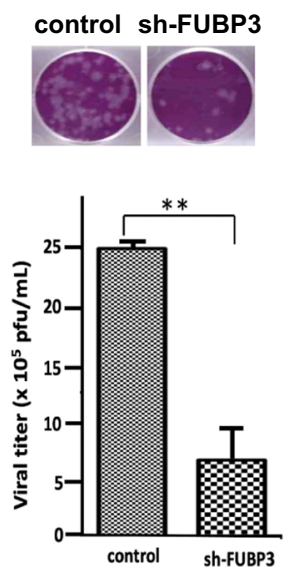

(E)

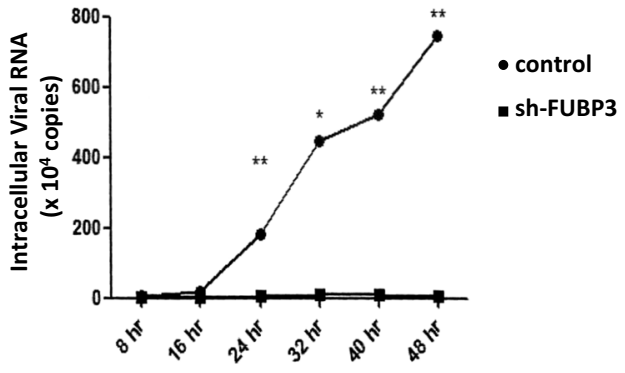

Fig. 3 FUBP3 regulated the viral protein translation and viral RNA replication. To establish the stable knocked down FUBP3 cell line, BHK-21 cells were transfected with shFUBP3 plasmid $(5 \mu \mathrm{g})$ for $72 \mathrm{~h}$, followed by selection with puromycin to obtain the FUBP3 stable knocked-down cells. The expression levels of FUBP3 was detected by Western blotting using anti-FUBP3 specific antibody. A $\beta$-actin expression was used as an internal control (a). The untreated BHK-21 cells (c) and sh-FUBP3-transfected cells $(\mathbf{b})$ were then infected with JEV $(\mathrm{MOI}=2)$ for 4, 8, 16, 24, 32, 40, $48 \mathrm{~h}$ and protein expression was analyzed by Western blotting using anti-NS specific antibody. A $\beta$-actin expression was used as an internal control. d Measurement of JE viral titers at $24 \mathrm{~h}$ post-infection from untreated and sh-FUBP3-transfected cells were performed by plaque forming assay. e The pCMV-FUBP3-Flag vector and pCMV-Flag vector $(8 \mu \mathrm{g})$ were then transfected in the stable knocked-down FBP3 cells for $24 \mathrm{~h}$, followed by infection with JEV $(\mathrm{MOI}=2)$ for 4, 8, 16, 24, 32, 40, $48 \mathrm{~h}$. The intracellular viral RNA production was then analyzed by RT-qPCR using specific primers as described in the Methods. $\left({ }^{*} p<0.05 ;{ }^{* *} p<0.01 ;{ }^{* *} p<0.001\right)$

overexpressed FUBP3 cells (merge images: Fig. 6f, j) was more pronounced than in JEV-infected cells alone (control group; Fig. 6k, l). The exogenous FUBP3 was also shown to move into the viral replication complex after JEV infection and to assist in viral replication.

\section{Discussion}

When an RNA virus infects a host cell, it requires the assistance of many host proteins for intracellular replication. The untranslated region of the viral RNA gene itself also plays a key role. In fact, the cyclization of the $3^{\prime}$ untranslated region ( $\left.3^{\prime} \mathrm{UTR}\right)$ is a very important mechanism. In addition to the cyclization of $3^{\prime} U T R$, this untranslated region also binds to some host proteins to promote/inhibit the replication of RNA viruses. In this study, using $3^{\prime}$ UTR labeled biotin, we identified four host proteins that bind to the $3^{\prime} \mathrm{UTR}$, namely GRP78, FUBP1, FUBP3 and hnRNP A1. GRP78 was reported to be present in the JEV-induced secretion medium and is participated in viral maturation [30]. FUBP1 acts as a defense host factor against JEV by inhibiting viral protein production [29]. Here, we demonstrated that FUBP3 regulated viral RNA replication of JEV and facilitated subsequent viral translation and viral particle production.

Many studies have reported that some host proteins are attracted to bind to the $3^{\prime}$ UTR of JEV after infection and affect viral replication or translation. For example, the La protein bound to the SL structure of the JEV $3^{\prime} \mathrm{UTR}$, unraveled the secondary structure of viral RNA, and promoted viral replication and translation [33]; DDX5 interacted with the JEV core protein, NS3 and NS5 proteins, moved into the cytoplasm during JEV infection, and bound to the JEV $3^{\prime}$ UTR to regulate viral replication and translation [34]. DDX3 interacted with JEV NS3 and NS5 proteins during JEV infection and bound to JEV $3^{\prime} \mathrm{UTR}$, deregulating viral translocation and affecting subsequent JEV RNA replication [35]; the polypyrimidine tract-binding protein (PTB) itself functions to assist in the splicing, export and translation of cellular mRNA [36]. In addition to host proteins that bound to the JEV $3^{\prime} \mathrm{UTR}$ 

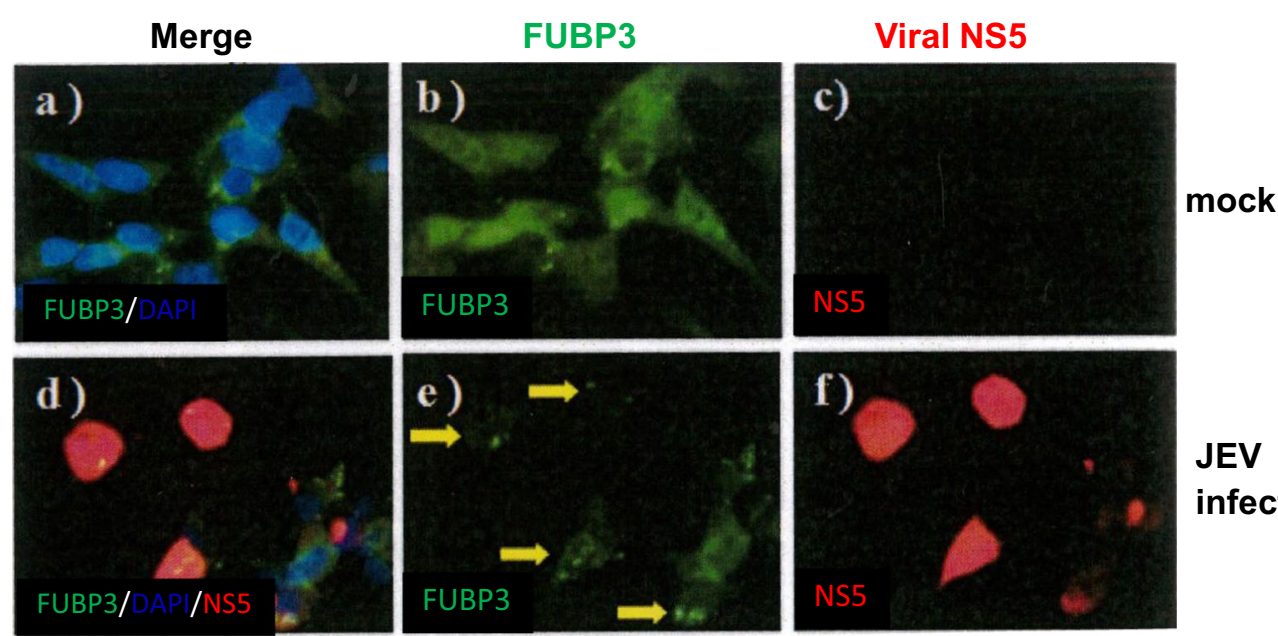

\section{JEV infection}

Fig. 4 Detection of colocalization of FUBP3 with JEV-NS5 protein in JEV-infected BHK-21 cells. Mock- or JEV-infected BHK-21 cells were harvested at $48 \mathrm{~h}$ post-infection and prepared for immunofluorescence analysis stained with antibodies that detect FUBP3 (green) and JEV-NS5 protein (red). Subcellular localization of FUBP3 (panel $\mathbf{b}, \mathbf{e}$ ) and viral NS5 protein (panel $\mathbf{f}$ ) in mock- and JEV-infected cells. The nucleus was stained with DAPI as shown in the merged image (panel $\mathbf{a}, \mathbf{c}$ ). Arrows showed the colocalization of FUBP3 with NS protein (panel e) were detected in the cytoplasm of JEV-infected cells. The Pearson correlation coefficient (PCC) of images of FITC-FUBP3 and Texas Red-NS5 is 0.9998 . Images are representative of three independent experiments that used three independent infections

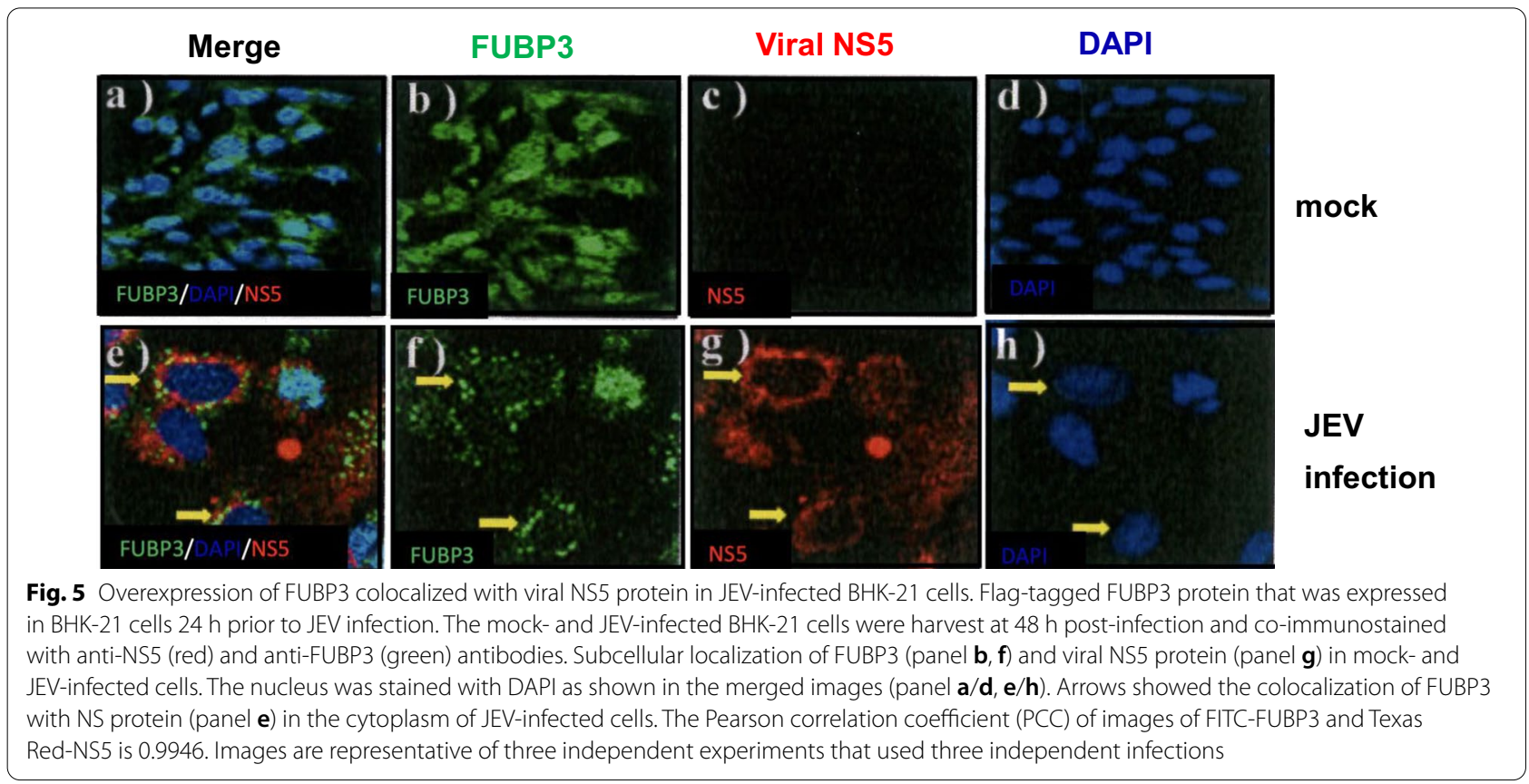

to positively regulate viral replication and translation, some host proteins bound to the JEV $3^{\prime} \mathrm{UTR}$ to inhibit viral replication, for example, FUBP1 bound to the JEV $5^{\prime}$ UTR and $3^{\prime}$ UTR to inhibit JEV protein translation, which in turn affected viral replication [29], and zinc-finger antiviral protein (ZAP) bound to the JEV $3^{\prime}$ UTR to inhibit viral protein translation and enhance viral RNA degradation. It also destabilized viral RNA by interacting with XRN1 and Rrp46 (EXOSC5), Rrp40 (EXOSC3) and Rrp42 (EXOSC7) [37]. We demonstrated that the FUBP3 protein can positively regulate the translation of JE viral proteins, and after JE virus infects cells, its intracellular location changes and accumulates into granules, which bind to the $3^{\prime}$ UTR of the virus gene and affect the 

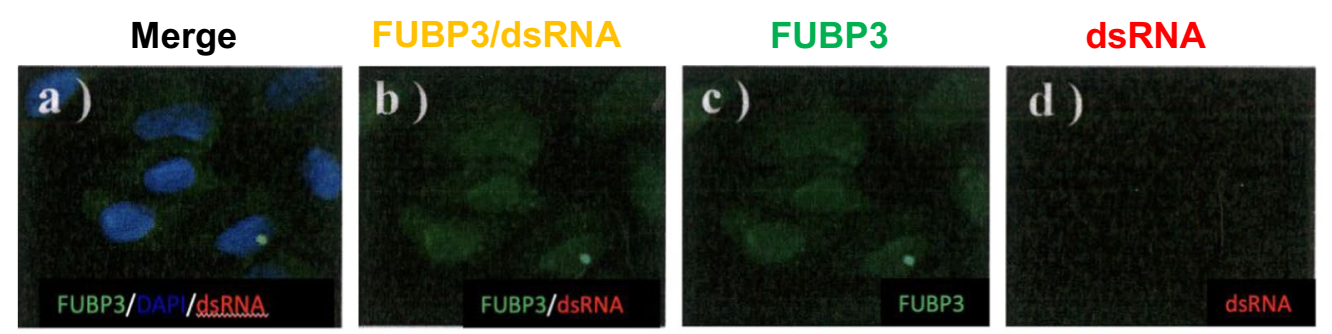

\section{mock}
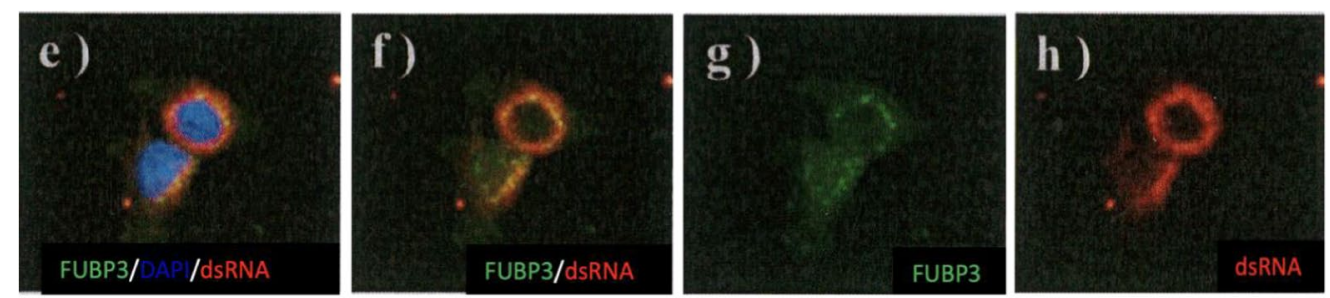

\section{JEV \\ infection}
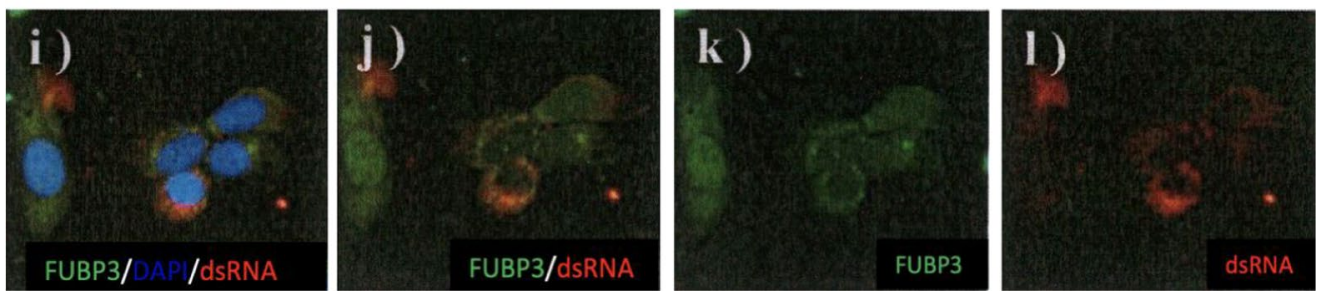

\section{JEV}

infection

Fig. 6 FUBP3 relocated to the JE replication complex. BHK-21 cells were first transfected with the flag-tagged FUBP3 expressing plasmid to overexpress the FUBP3 protein and then proceeded to JEV infection. The mock-and JEV-infected BHK-21 cells were harvest at $48 \mathrm{~h}$ post-infection and co-immunostained with anti-dsRNA (red) and anti-FUBP3 (green) antibodies. Panel $\mathbf{a}-\mathbf{d}$ are mock groups. Panel $\mathbf{e}-\mathbf{h}$ and $\mathbf{i}-\mathbf{I}$ are JEV infection groups (Images were taken from three independent experiments, and two representative groups were selected). Positive stained for dsRNA was only observed in the JEV-infected cells (panel $\mathbf{h}$, i). The nucleus was stained with DAPI as shown in the images (panel $\mathbf{a}, \mathbf{e}, \mathbf{f}$ ). The colocalization of FUBP3 with viral dsRNA protein (panel $\mathbf{f}, \mathbf{j}$ ) was detected in the cytoplasm of JEV-infected cells. The Pearson correlation coefficient (PCC) of images of FITC-FUBP3 and Texas Red-dsRNA is 0.9445 . The Images are representative of three independent experiments that used three independent infections

translation of the virus, and even further affect the replication of the virus.

FUBP1 and FUBP3 are known to bind to RNA and regulate the replication of certain viruses; for example, FUBP1 was used as an IRES transactivator, competing with FUBP2 for binding to the IRES structure of EV-A71, increasing IRES activity and promoting IRES-mediated translation [38]. As for HCV infection, FUBP1 moved from the cell to the cytoplasm and bound specifically to the poly $(\mathrm{U})$ structure in the poly(U/UC) domain of the HCV 3'UTR, promoting HCV viral replication [39]. In contrast, FUBP1 was reported negatively regulating viral infection, as FUBP1 moved from the nucleus to the cytoplasm during the early stages of JEV infection and bound specifically to the JEV $5^{\prime}$ UTR and $3^{\prime}$ UTR to inhibit viral replication. The UTR inhibited viral proteins or host proteins to assist viral translocation, further leading to reduced viral replication and affecting viral infection [29]. In contrast to FUBP1 and FUBP2, few reports have mentioned the functional relevance of FUBP3 for RNA viruses. Huang et al. showed that FUBP3 redistributed among subcellular compartments after EV-A71 infection and bound to the $5^{\prime} \mathrm{UTR}$ of EV-A71 to promote viral replication [28]. In this study, we used RNA pull down assay to identify both FUBP1 and FUBP3 in the JEV-infected BHK-21 cells. FUBP1 has been reported to bind to the UTR of JEV to inhibit JEV protein translation, while our results confirmed that FUBP3 could promote the replication of JEV to increase the viral titers. Previous reports have shown that both FUBP1 and FUBP2, which are also members of the FUBPs family, can bind to the IRES structure of EV-A71 and play the role of ITAFS, positively and negatively regulating the viral protein translocation of EV71, which in turn affects the viral replication [38]. In our study, we demonstrated that the FUBP3 protein can positively regulate the translation of JE viral proteins, and after JE virus infects cells, its intracellular location changes and accumulates into granules. Although both FUBP1 and FUBP3 can bind to the 3'UTR of JEV, the two proteins do not have the same ability to bind, and at the same time, the other host proteins interacting with each of the two proteins are also different. When the cells were 
infected by JEV, it affected the different levels of expression of various host proteins in the cells, which indirectly interfered with the degree of binding of FUBP1 and FUBP3 to the $3^{\prime} U T R$ of JEV, resulting in the different performance of the two proteins binding to the $3^{\prime} \mathrm{UTR}$ and affecting virus replication. It is possible that the region where the viral $3^{\prime} \mathrm{UTR}$ is bound competes with FUBP1 and other host factors that inhibit viral replication. In summary, when JEV infects the host cells, FUBP3 in the host cells will accumulate from the original intracellular even distribution into granules, and these granules may be in the same position as the dsRNA of JEV, playing the role of positive regulation of virus replication.

\section{Conclusion}

In the present study, we demonstrated that FUBP3 bound to the $3^{\prime}$ untranslated region of JEV and acted as a host factor to promote viral translation, and accompanied the viral replication complexes to accelerate viral RNA replication during the virus life cycle. Knockdown of the FUBP3 protein reduced viral infectivity by disrupting the JEV 3'-UTR-binding host factors. Therefore, inhibition of FUBP3 may be a novel approach to develop effective treatments and prevention strategies for JEV virus infection.

\section{Abbreviations \\ FUSE: Far upstream element; FUBP: Far upstream element-binding protein; JEV: Japanese encephalitis virus; UTR: Untranslated region; UAR: Upstream of AUG region; DENV: Dengue virus; WNV: West Nile virus; MOl: Multiplicity of infection.}

\section{Acknowledgements}

The authors would like to thank Dr. Robert YL Wang from Chang Gung University for providing the FUBP3-specific siRNA as well as the JEV NS5 specific antibody. The authors also thanks Dr. David Pledger and Mr. Valerio Puggioni for carefully editing this revised manuscript.

\section{Authors' contributions}

YMC conceived of the study, and drafted the manuscript. HHC and PX carried out the virological and biochemical assays and drafted the manuscript. PX and WT participated in the design of the study. YMC participated in the design and the drafting of the manuscript. All authors read and approved the final manuscript.

\section{Funding}

This research was supported in part by a grant from the Ministry of Science and Technology (MOST 106-2313-B-019-009), and the Center of Excellence for the Oceans, National Taiwan Ocean University, which is sponsored by the Ministry of Education, Taiwan.

\section{Availability of data and materials}

The datasets used and analyzed during the current study are available from the corresponding author on reasonable request.

\section{Declarations}

Ethics approval and consent to participate

Not applicable.

\section{Consent to publication}

Not applicable.

\section{Competing interests}

The authors declare that they have no competing interests.

\section{Author details}

'Xiangyang No.1 People's HospitalHubei University of Medicine, Xiangyang, Hubei Province, China. ${ }^{2}$ Department of Clinical Laboratory, Xiangyang No.1 People's Hospital, Hubei University of Medicine, Xiangyang, China. ${ }^{3}$ Bachelor Degree Program in Marine Biotechnology, College of Life Sciences, National Taiwan Ocean University, Keelung 20224, Taiwan. ${ }^{4}$ Center of Excellence for the Oceans and Matsu Marine Research Center, National Taiwan Ocean University, Keelung 20224, Taiwan.

Received: 18 August 2021 Accepted: 10 November 2021 Published online: 18 November 2021

\section{References}

1. Misra UK, Kalita J. Overview: Japanese encephalitis. Prog Neurobiol. 2010;91:108-20.

2. Chambers TJ, Hahn CS, Galler R, Rice CM. Flavivirus genome organization, expression, and replication. Annu Rev Microbiol. 1990;44:649-88.

3. Markoff L. 5'-and $3^{\prime}$-noncoding regions in flavivirus RNA. Adv Virus Res. 2003;59:177-288.

4. Heinz FX, Allison SL. Flavivirus structure and membrane fusion. Adv Virus Res. 2003;59:63-99.

5. Rice CM. Flaviviridae: the viruses and their replication. In: Fields BN, Knipe DM, Howley PM, editors. Fields virology. Philadelphia: Lippincott-Raven; 1996. p. 931-59.

6. Bidet K, Garcia-Blanco MA. Flaviviral RNAs: weapons and targets in the war between virus and host. Biochem J. 2014;462:215-30.

7. Alvarez DE, Ezcurra ALDL, Fucito S, Gamarnik AV. Role of RNA structures present at the $3^{\prime}$ UTR of dengue virus on translation, RNA synthesis, and viral replication. Virology. 2005;339:200-12.

8. Khromykh A, Westaway E. RNA binding properties of core protein of the flavivirus Kunjin. Arch Virol. 1996;141:685-99.

9. Hahn CS, Hahn YS, Rice CM, Lee E, Dalgarno L, Strauss EG, Strauss JH. Conserved elements in the $3^{\prime}$ untranslated region of flavivirus RNAs and potential cyclization sequences. J Mol Biol. 1987;198:33-41.

10. Alvarez DE, Filomatori CV, Gamarnik AV. Functional analysis of dengue virus cyclization sequences located at the $5^{\prime}$ and $3^{\prime}$ UTRs. Virology. 2008;375:223-35

11. Friebe P, Harris E. Interplay of RNA elements in the dengue virus $5^{\prime}$ and $3^{\prime}$ ends required for viral RNA replication. J Virol. 2010;84:6103-18.

12. Yun S-I, Choi Y-J, Song B-H, Lee Y-M. $3^{\prime}$ Cis-acting elements that contribute to the competence and efficiency of Japanese encephalitis virus genome replication: functional importance of sequence duplications, deletions, and substitutions. J Virol. 2009;83:7909-30.

13. Uchil PD, Satchidanandam V. Characterization of RNA synthesis, replication mechanism, and in vitro RNA-dependent RNA polymerase activity of Japanese encephalitis virus. Virology. 2003;307:358-71.

14. Polacek C, Friebe P, Harris E. Poly (A)-binding protein binds to the nonpolyadenylated $3^{\prime}$ untranslated region of dengue virus and modulates translation efficiency. J Gen Virol. 2009;90:687-92.

15. Benjamin LR, Chung HJ, Sanford S, Kouzine F, Liu J, Levens D. Hierarchical mechanisms build the DNA-binding specificity of FUSE binding protein. Proc Natl Acad Sci USA. 2008;105:18296-301.

16. Chung HJ, Liu J, Dundr M, Nie Z, Sanford S, Levens D. FBPs are calibrated molecular tools to adjust gene expression. Mol Cell Biol. 2006;26:6584-97.

17. Gherzi R, Chen CY, Ramos A, Briata P. KSRP controls pleiotropic cellular functions. Semin Cell Dev Biol. 2014;34:2-8.

18. Li X, Lin WJ, Chen CY, Si Y, Zhang X, Lu L, Suswam E, Zheng L, King PH. KSRP: a checkpoint for inflammatory cytokine production in astrocytes. Glia. 2012;60:1773-84.

19. Zhang J, Chen QM. Far upstream element binding protein 1: a commander of transcription, translation and beyond. Oncogene. 2013;32:2907-16. 
20. Duncan R, Collins I, Tomonaga T, Zhang T, Levens D. A unique transactivation sequence motif is found in the carboxyl-terminal domain of the single-strand-binding protein FBP. Mol Cell Biol. 1996;16:2274-82.

21. Bazar L, Harris V, Sunitha I, Hartmann D, Avigan M. A transactivator of c-myc is coordinately regulated with the proto-oncogene during cellular growth. Oncogene. 1995;10:2229-38.

22. Wang X, Avigan M, Norgren RB Jr. FUSE-binding protein is developmentally regulated and is highly expressed in mouse and chicken embryonic brain. Neurosci Lett. 1998;252:191-4.

23. Kim MJ, Park B-J, Kang Y-S, Kim HJ, Park J-H, Kang JW, Lee SW, Han JM, Lee H-W, Kim S. Downregulation of FUSE-binding protein and c-myc by tRNA synthetase cofactor p38 is required for lung cell differentiation. Nat Genet. 2003;34:330-6.

24. Williams BY, Hamilton SL, Sarkar HK. The survival motor neuron protein interacts with the transactivator FUSE binding protein from human fetal brain. FEBS Lett. 2000;470:207-10.

25. Bouchireb N, Clark M. Human gene mapping report. Human FUSE binding protein 3 gene (FBP3). Map position 9q33-341. Chromosome Res. 1999;7:577.

26. Davis-Smyth T, Duncan RC, Zheng T, Michelotti G, Levens D. The far upstream element-binding proteins comprise an ancient family of singlestrand DNA-binding transactivators. J Biol Chem. 1996;271:31679-87.

27. Weber A, Kristiansen I, Johannsen M, Oelrich B, Scholmann K, Gunia S, May M, Meyer H-A, Behnke S, Moch H. The FUSE binding proteins FBP1 and $\mathrm{FBP} 3$ are potential c-myc regulators in renal, but not in prostate and bladder cancer. BMC Cancer. 2008;8:1-11.

28. Huang HI, Chang YY, Lin JY, Kuo RL, Liu HP, Shih SR, Wu CC. Interactome analysis of the EV71 $5^{\prime}$ untranslated region in differentiated neuronal cells $\mathrm{SH}-\mathrm{SY} 5 \mathrm{Y}$ and regulatory role of FBP3 in viral replication. Proteomics. 2016;16:2351-62.

29. Chien HL, Liao CL, Lin YL. FUSE binding protein 1 interacts with untranslated regions of Japanese encephalitis virus RNA and negatively regulates viral replication. J Virol. 2011:85:4698-706.

30. Wu YP, Chang CM, Hung CY, Tsai MC, Schuyler SC, Wang RY. Japanese encephalitis virus co-opts the ER-stress response protein GRP78 for viral infectivity. Virol J. 2011;8:128.
31. Kuo R-L, Kao L-T, Lin S-J, Wang RY-L, Shih S-R. MDA5 plays a crucial role in enterovirus 71 RNA-mediated IRF3 activation. PLOS ONE. 2013;8:431.

32. Dixit U, Pandey AK, Liu Z, Kumar S, Neiditch MB, Klein KM, Pandey VN. FUSE binding protein 1 facilitates persistent hepatitis $C$ virus replication in hepatoma cells by regulating tumor suppressor p53. J Virol. 2015:89:7905-21.

33. Vashist S, Anantpadma M, Sharma H, Vrati S. La protein binds the predicted loop structures in the $3^{\prime}$ non-coding region of Japanese encephalitis virus genome: role in virus replication. J Gen Virol. 2009;90:1343-52.

34. Li C, Ge L, Li P, Wang Y, Sun M, Huang L, Ishag H, Di D, Shen Z, Fan W. The DEAD-box RNA helicase DDX5 acts as a positive regulator of Japanese encephalitis virus replication by binding to viral 3' UTR. Antiviral Res. 2013;100:487-99.

35. Li C, Ge L, Li P, Wang Y, Dai J, Sun M, Huang L, Shen Z, Hu X, Ishag H. Cellular DDX3 regulates Japanese encephalitis virus replication by interacting with viral un-translated regions. Virology. 2014;449:70-81.

36. Kim SM, Jeong YS. Polypyrimidine tract-binding protein interacts with the 3' stem-loop region of Japanese encephalitis virus negative-strand RNA. Virus Res. 2006;115:131-40.

37. Chiu H-P, Chiu H, Yang C-F, Lee Y-L, Chiu F-L, Kuo H-C, Lin R-J, Lin Y-L. Inhibition of Japanese encephalitis virus infection by the host zinc-finger antiviral protein. PLoS Pathog. 2018;14:e1007166.

38. Huang P-N, Lin J-Y, Locker N, Kung Y-A, Hung C-T, Lin J-Y, Huang H-I, Li $M-L$, Shih S-R. Far upstream element binding protein 1 binds the internal ribosomal entry site of enterovirus 71 and enhances viral translation and viral growth. Nucleic Acids Res. 2011;39:9633-48.

39. Zhang Z, Harris D, Pandey VN. The FUSE binding protein is a cellular factor required for efficient replication of hepatitis $C$ virus. J Virol. 2008;82:5761-73

\section{Publisher's Note}

Springer Nature remains neutral with regard to jurisdictional claims in published maps and institutional affiliations.
Ready to submit your research? Choose BMC and benefit from:

- fast, convenient online submission

- thorough peer review by experienced researchers in your field

- rapid publication on acceptance

- support for research data, including large and complex data types

- gold Open Access which fosters wider collaboration and increased citations

- maximum visibility for your research: over 100M website views per year

At BMC, research is always in progress.

Learn more biomedcentral.com/submissions 